\title{
Performance Improvement of WSNs using Joint Reed Solomon and Network Coding
}

\author{
Khalifa A. Salim, PhD \\ Asst. Prof. \\ ICE Engineering Department \\ Baghdad University \\ Baghdad, Iraq
}

\author{
Maryam K. Abboud \\ Student Member, IEEE \\ Computer Engineering Department \\ Al-Farabi University \\ Baghdad, Iraq
}

\begin{abstract}
In this paper, a combined error control coding and network coding (NC) scheme is proposed for performance improvement of wireless sensor networks (WSNs) in terms of throughput and Bit error rate (BER). A suggested network model is conserved in simulations, which integrates the characteristics of; mesh, stare, and tree network models with eleven nodes. Reed-Solomon (RS) code is considered as a Forward Error Control (FEC) coding scheme in simulations. The simulation results shows that BER may slightly increases using NC, while at high Signal to Noise power Ratios (SNRs), throughput performance is improved by about 64\%. This improvement is obtained even when the transmission experience a frequency selective fading environments.
\end{abstract}

\section{Keywords}

NC, RS code, WSN, Channel Coding.

\section{INTRODUCTION}

WSN consists of a number of small, autonomous devices called Wireless Sensor nodes, which formed with number of sensors integrated with tiny microprocessors. Many applications where WSN nodes adopted and working in a cooperative manner [1]. The fundamental characteristic of WSNs is the multi-hop transmission which affected by the noisy links and hence effects the throughput performance of the whole network [2]. Hence, to cover this problem, FEC is adopted to reduce transmission errors, while $\mathrm{NC}$ is used for required transmissions reduction. FEC and $\mathrm{NC}$ was covered by many researchers in this field with different conditions. A NC scheme was introduced by [3], where a relay channel mode used to perform $\mathrm{NC}$ between two users and the users perform channel coding operations. $\mathrm{NC}$ was combined with FEC coding in [4], where channel decoding is performed after $\mathrm{NC}$ at the destination by combined soft/hard decisions. In [58], system robustness increased by applying RS and NC schemes at intermediate nodes of a WSN, where system robustness enhanced and the require resources was reduced.
The same combination was proposed in [9], where it adopted for star network model. In the proposed system, Automatic Repeat Request (ARQ) has been adopted as a FEC code combined with $\mathrm{NC}$ which integrates multiple packets to form one packet to be transmitted. The QoS for WSNs can be achieved by the optimal selection for the relay nodes which combines NC with FEC code after parameters adjustment as proposed by [10-13]. A generalized WSN model combines star, mesh and tree structures is considered in this paper, WSN nodes are classified into; source nodes, coding nodes, and destination nodes. A node to node error correction encoding/decoding scenario with $\mathrm{NC}$ was performed in an end to end manner (encoding/decoding) by the suggested system. At the source nodes, before data sending through the network, packets are FEC encoded with RS code, where they reprocessed at intermediate nodes and RS decoded to be combined using NC. After NC process, the network coded packets are recoded using RS code. At the destinations side, to recover the original data, RS decoding is implemented with $\mathrm{NC}$ decoding for packets received from different links. In this paper; system model and parameters are proposed in sections 2 and 3. Performance evaluation, in sections 4. Finally, main conclusions and suggestions for future work is proposed in section 5 .

\section{SYSTEM MODEL}

The proposed system is shown in Figure 1, where the combination takes place. Binary Phase Shift Keying (BPSK) is adopted for modulation and detection. In FEC coding stage, $\mathrm{RS}(n, k, t)$ code is considered, which provides maximum separable property [14]. In the presence work, the code word length $n$ and the data block length $k$ are the two important RS code parameters needed to be defined. The correction capability $t$ of $\mathrm{RS}$ code used is $t=(n-k) / 2$ [14] Berlekamp and Chien's algorithms are used for RS decoding [15]. $(255,247,4)$ representing RS coding parameters $(n, k, t)$ considered in this work. 


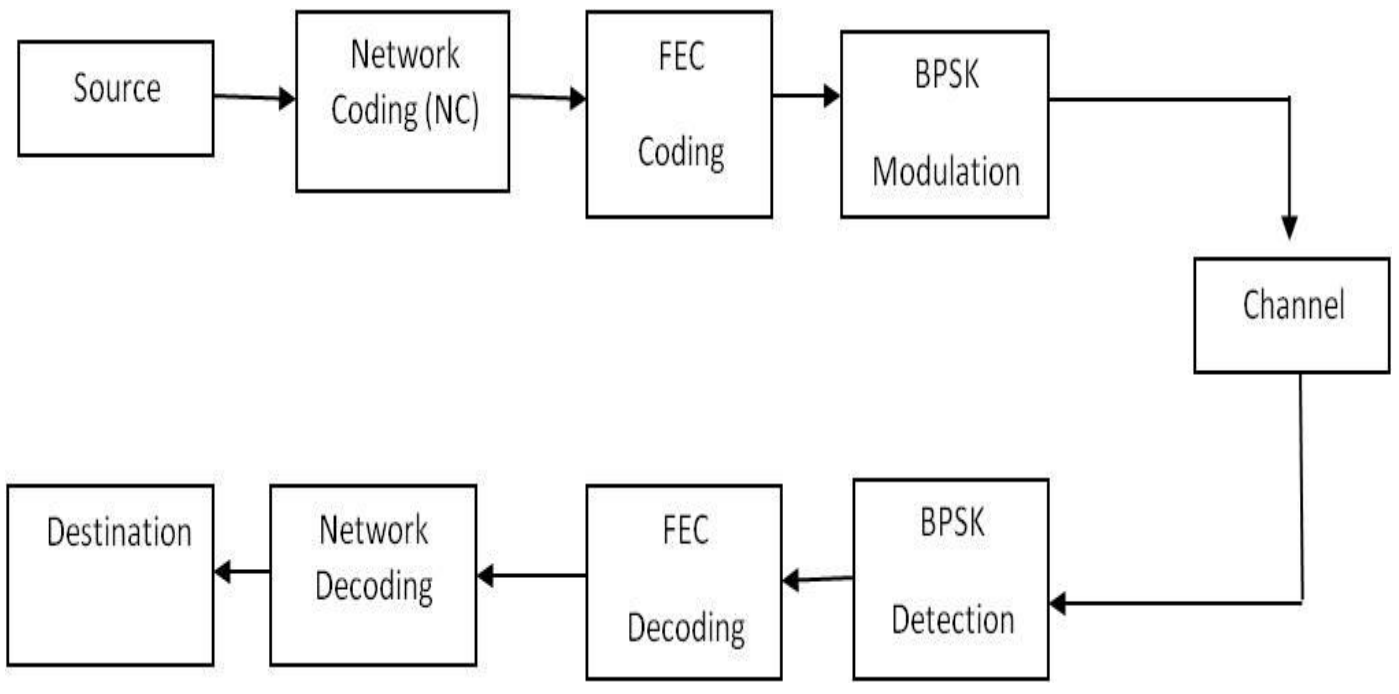

Fig. 1: Proposed System Model

Based on generating independent vectors [16], a Random Linear Network Coding (RLNC) was adopted at NC block of Figure 1 at each coding node. Network encoding in performed at coding nodes, while decoding is performed at sink node. A First in First out (FIFO) queue is used to handle the received packets to be coded using $\mathrm{NC}$, where system throughput improved. For network coding, packets are linearly combined using independent vectors generated with elements related to a Galois Field (GF), where NC process follows equation (1) [17];

$Y_{k}=\sum_{i=1}^{m} p_{i, k} R_{k}^{i}$

$R^{i}$ : Source packets $\left(R^{1} \ldots \ldots \ldots R^{m}\right)$

$p_{i}$ : Coding vectors $\left(p_{1} \ldots \ldots p_{m}\right)$ of $F_{2}^{m}$ elements

$X$ : Coded packet.

$Y_{k}$ and $R_{k}^{i}: k^{\text {th }}$ symbols of $\mathrm{Y}$ and $R^{i}$ respectively.

A simple Gaussian elimination applied for the purpose of NC decoding using encoding vectors attached in packet's header [7], [8], [10], [18]. AWGN is the Additive White Gaussian Noise considered in simulations along with; flat fading and a multipath selective channels with moderate level of distortion, where its parameters are shown in Table 1 [19].

Table 1. Multipath Channel Model [19].

\begin{tabular}{|c|c|c|}
\hline Taps No. & Relative Delay $(\boldsymbol{\mu s})$ & Average Power $(\mathbf{d B})$ \\
\hline 1 & 0 & 0 \\
\hline 2 & 0.4 & -5 \\
\hline 3 & 0.9 & -10 \\
\hline
\end{tabular}

\section{PROPOSED NETWORK MODEL}

In this work, a network model is proposed which enables cooperative communication among its nodes in order to achieve the advantage of combining $\mathrm{NC}$ with channel coding. The vague nature of WSN nodes distribution makes performance evaluation to be a critical point especially for large networks. Thus, the proposed network model in this paper can be assumed to be a more generalized model which integrates mesh, star, and tree network model's properties in one model as shown in Figure 2.

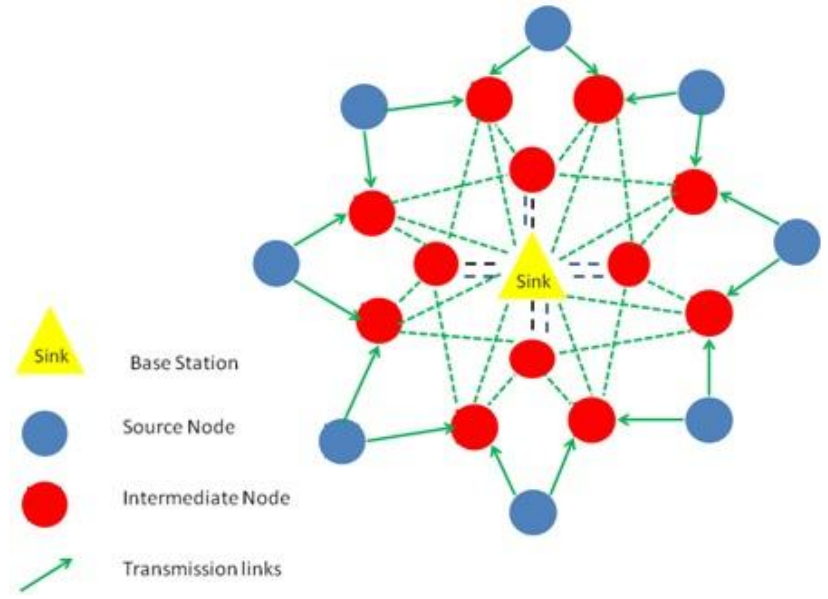

Fig. 2: Proposed Network Model.

The network of Figure 2 consists of a few number of source, intermediate, and a base station nodes, where it forms an elementary sub-network belonging to a large WSN. The intermediate nodes acts as the coding nodes in the network, while the base station is the sink node. Data packets are generated at the source nodes where FEC encoding applied. NC and FEC coding/decoding are performed at the intermediate nodes. At the other hand, FEC and NC decoding processes are carried out at the final destination node. Figure 3 represents the processing block of all node types; source, intermediate and sink nodes. In Figure 3, there is no need to perform NC decoding process at the coding nodes since the original data is requested by the final recipient. 


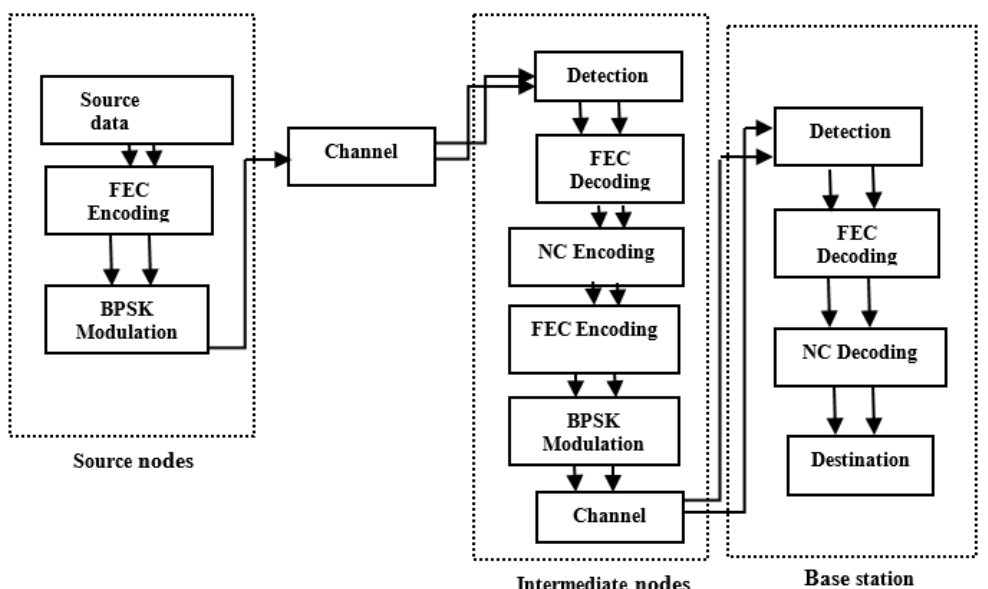

Fig. 3: Processing Blocks of Different Nodes.

\section{SIMULATION TESTS AND RESULTS}

In the proposed work, two test conditions are used to measure Bit Error Rate (BER) and Throughput (Thru); RS coding without NC process and RS coding in the presence of NC process. BER and Throughput are calculated by the following equations ( 2 and 3 );

$$
\text { BER }=\frac{\text { Total number of erroneous received bits }}{\text { Total number of transmitted bits }}
$$

To measure throughput performance, two cases must be taken into account; first, throughput measuring without NC process and the second is throughput with $\mathrm{NC}$ process to be compared with the first one.

$$
\mathrm{Thru}_{\text {without } N C}=\frac{\text { Total number of correct received bits }}{\text { Total number of transmitted bits }}
$$

In the presence of $\mathrm{NC}$, the most effective parameter in calculating the throughput of the network is defined as NC gain, which represents the gain achieved in transmissions reduction as the basic $\mathrm{NC}$ advantages. Hence;

$\mathrm{Thru}_{\text {with } N C}=\frac{\text { Total number of correct received bits }}{\text { Total number of transmitted bits }} \times N C_{\text {gain }}(4)$

Where, NC gain is defined by;

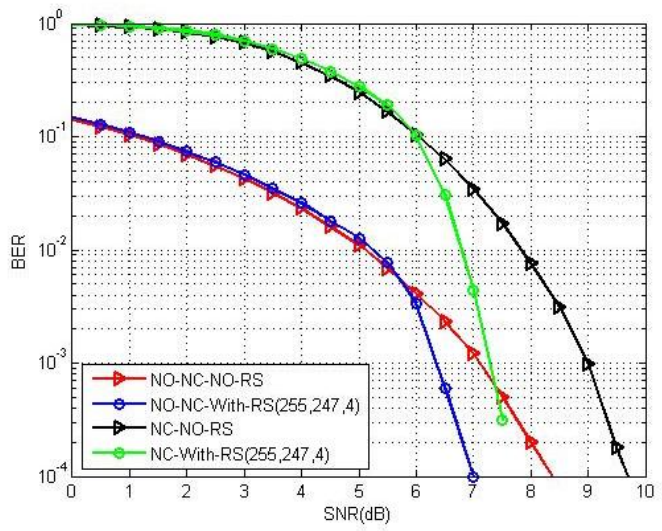

(a)
$N C_{\text {gain }} \frac{\text { Total number of transmissions without NC }}{\text { Total number of transmissions with NC }}$

Another important parameter used to measure the performance gain in system throughput is given by equation (6);

Throughput gain $=\frac{\text { new throughput-old throughput }}{\text { new throughput }} \times 100 \%$

In this work, four main scenarios are considered:

1- Transmission without coding.

2- Transmission without NC, with RS coding.

3- Transmission with NC, without RS coding.

4- Transmission with NC and RS coding.

In simulations, link's capacity is assumed to be $\left(1 \frac{\text { packet }}{\text { unit time }}\right)$. The transmission considers $4 \mathrm{Kbps}$ transmission rate, where all intermediate nodes performs NC. The BER performance of the proposed network is shown in Figure 4, assuming the combination of RS of $(255,247,4)$ specifications with NC over various channels, and throughput performance is presented in Figure 5.

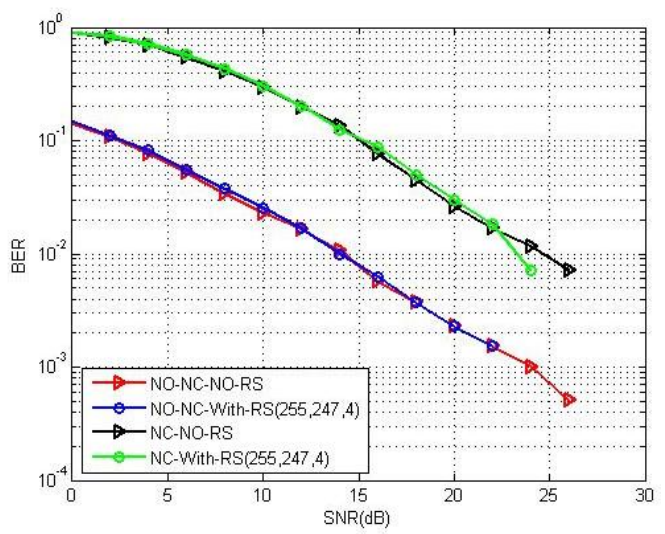

(b) 


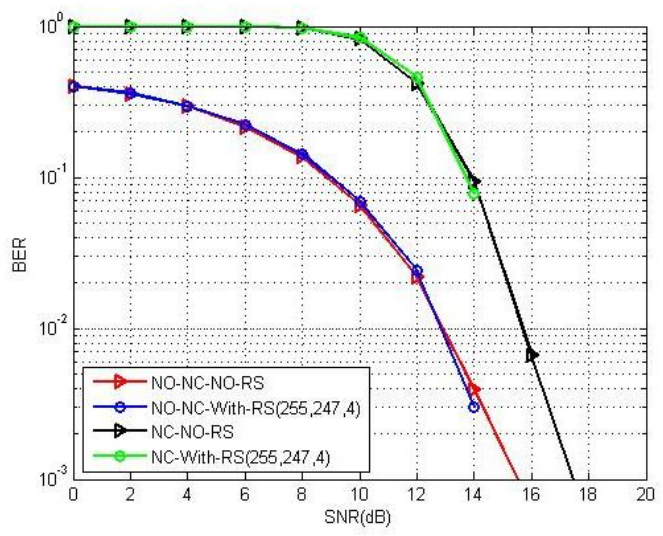

(c)

Fig. 4: BER performance of the proposed network considering various channel models.

a- AWGN b- Flat Fading

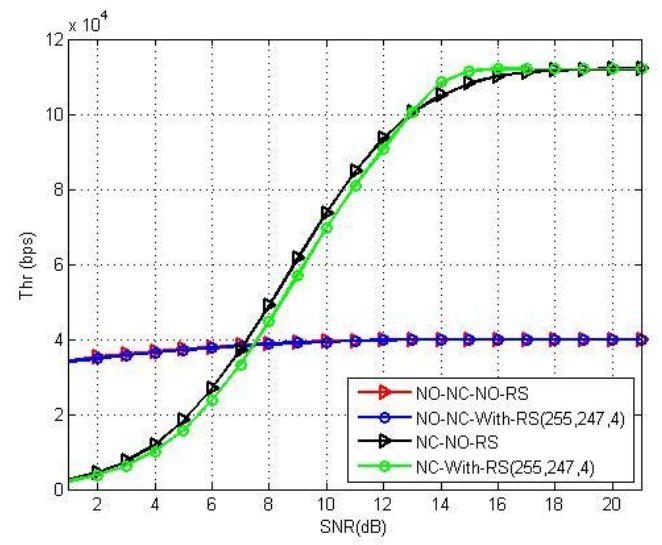

(a) c- Multipath Fading

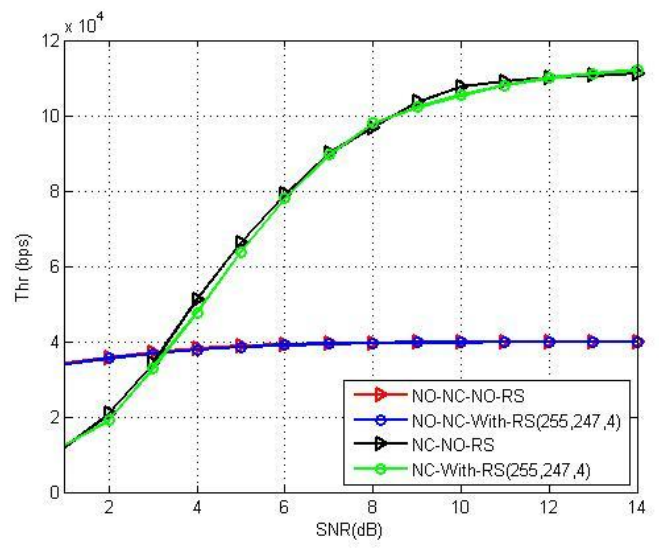

(b)

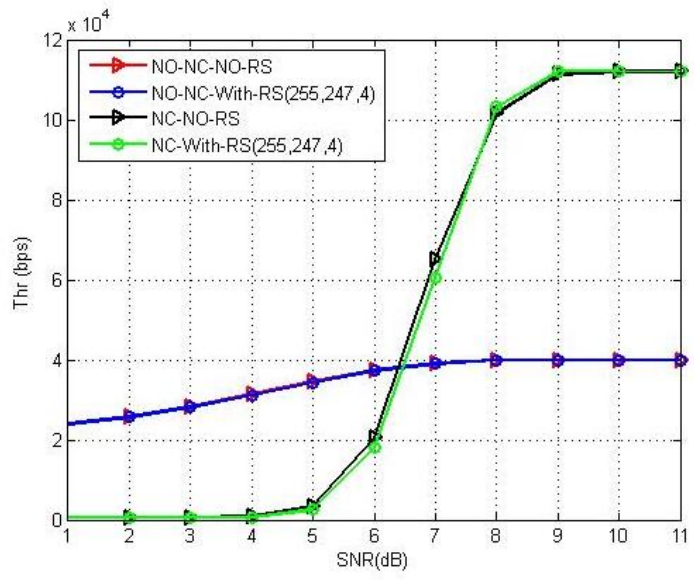

(c)

Fig. 5: Throughput performance of the proposed network considering various channel models.

\section{a- AWGN b- Flat Fading c- Multipath Fading}

As could be noticed, the proposed combination results a transmissions reduction, where 10 transmissions are needed for packet delivering to the end recipients, while 28 transmissions are required in the case where there is no $\mathrm{NC}$ applied. This results in $64 \%$ transmission throughput gain. At the other hand, a low SNR values are required in order to obtain high throughputs. Table 2 summarizes this benefit over different wireless channel environments, where it shows that as transmission throughput improved from $40 \mathrm{Kbps}$ to $117 \mathrm{Kbps}, \mathrm{NC}$ addition reduces the required SNR from $20 \mathrm{~dB}$ over AWGN channel to $11 \mathrm{~dB}$ over multipath fading channel. 
Table 2. Required SNR for Throughput Improvement with NC over Different Channel Environments

\begin{tabular}{|c|c|c|c|c|c|}
\hline \multicolumn{2}{|c|}{ AWGN } & \multicolumn{2}{c|}{ Flat Fading } & \multicolumn{2}{c|}{ Multipath Fading } \\
\hline \multicolumn{2}{|c|}{$20 \mathrm{~dB}$} & \multicolumn{2}{|c|}{$14 \mathrm{~dB}$} & \multicolumn{2}{c|}{$11 \mathrm{~dB}$} \\
\hline NC & No NC & NC & No NC & NC & No NC \\
\hline 117 & 40 & 117 & 40 & 117 & 40 Kbps \\
Kbps & Kbps & Kbps & Kbps & Kbps & \\
\hline
\end{tabular}

Regarding BER performance over AWGN channel, an improvement achieved by about $1.7 \mathrm{~dB}$ in SNR by applying RS coding in the presence of NC. And by testing the network performance when there is no NC applied, the addition of RS code improves the performance by about $0.7 \mathrm{~dB}$. Regarding multipath and flat fading channels, the BER performance is decayed clearly at low SRN values of a fading environments when adopting NC process. At the other hand, its addition improves BER at high SNR by about $2 \mathrm{~dB}$ over multipath fading channel at a BER of $10^{-3}$ as compared to the BER degradation appears at low SRN values.

\section{CONCLUSIONS}

In this paper, FEC and NC combinations are tested considering a generalized network model. An improvement in BER performance by combining RS coding with $\mathrm{NC}$ was achieved with a considerable reduction in number of transmissions which improves system throughput. For BER performance tests, the SNR gain over AWGN channel is relatively grater as compared to that over fading channels, while throughput improvement tests shows that the required SNR is reduced especially in multipath fading channels, where $117 \mathrm{Kbps}$ was achieved using $\mathrm{NC}$ as compared to 40 Kbps for the case where no NC used. Finally, NC with RS combination can provide an increasing in throughput on a cost of slight BER degradation at low SNR values.

\section{REFERENCES}

[1] H. Karl and A. Willig, "Protocols and Architectures for Wireless Sensor Networks", John Wiley and Sons, Ltd, Sussex, England, 2005.

[2] Z. Hu, B. Li, "Fundamental performance limits of wireless sensor networks", Journals of ad Hoc and sensor networks AHSWN, 2004.

[3] C. Hausl and J. Hagenauer, "Iterative Network and Channel Decoding for the Two-Way Relay Channel", in Proceedings IEEE International Conference on Communications, Vol. 4, Istanbul, June 2006.

[4] C. Hausl and P. Dupraz, "Joint Network-Channel Coding for the Multiple-Access Relay Channel", IEEE Communications Society on Sensors and Ad Hoc Communications and Networks, Vol. 3, Reston, Virginia, Sept. 2006.

[5] C. Hausl, "Joint Network-Channel Coding for Wireless Relay Networks", College of Electrical Engineering and Information Technology, Technical University of Munich, Munich, Nov. 2008.

[6] Z. Guo, J. Huang, B. Wang, J. Cui, S. Zhou, and W. P., "Non-binary joint network and channel coding for underwater sensor networks", The International Workshop on Under Water Networks, September 2008.

[7] Z. Guo, J. Huang, B. Wang, S. Zhou, J.-H. Cui and P. Willett "A Practical Joint Network-Channel Coding Scheme for Reliable Communication in Wireless Networks", in Proceedings of IEEE Transactions on wireless communications, Vol.11, Issue 6, June 2012.

[8] T. Matsuda and T. Takine. "Multicast Communications with Reed Solomon/Network Joint Coding in Wireless Multi-hop Networks", Journal of Communications, Vol. 4, No 11, Dec. 2009

[9] S. J. Johnson, L. Ong, and C. M. Kellett, "Joint Channel-Network Coding Strategies for Networks with Low Complexity Relays", European Transactions on Telecommunications, Vol. 22, Issue 7, Oct. 2011.

[10] C. Koller, M. Haenggi, J. Kliewer, D. J. Costello, J. L. Fellow, "Joint Design of Channel and Network Coding for Star Networks connected by Binary Symmetric Channels", IEEE Transactions on Communications, Vol. 62, Issue 1, Nov. 2013.

[11] G. Angelopoulos, A. Paidimarri, A. P. Chandrakasan and M. Medard, "Experimental Study of the Interplay of Channel and Network Coding in Low Power Sensor Applications", IEEE International Conference on Communications (ICC), Budapest, June 2013.

[12] Y. Jin and P. Ruan, "Adaptive Cooperative FEC Based on Combination of Network Coding and Channel Coding for Wireless Sensor Networks", Journal of networks, Vol. 9, No. 2, Feb. 2014.

[13] D. C. Adams, J. Du, M. Medard and C. C. Yu, "Delay Constrained Throughput-Reliability Tradeoff in Network-Coded Wireless Systems", IEEE Global Communications Conference (GLOBCOM), Austin, Texas, Dec. 2014.

[14] J. Moreira and P. Farrell, "Essentials of Error Control Coding", John Wiley and Sons Ltd, West Sussex, England, 2006.

[15] X. T. Vu, P. Duhamel, and M. D. Renzo, "Performance Analysis of Network Coded Cooperation with Channel Coding and Adaptive DF-Based Relaying in Rayleigh Fading Channels", IEEE Signal Processing Letters, Institute of Electrical and Electronics Engineers (IEEE), Vol. 22, No. 9, Mar. 2015.

[16] P. Chou and Y. Wu, " Network Coding for the Internet and Wireless Networks", IEEE Signal Processing Magazine, Volume 24, Issue 5, June 2007.

[17] S. Katti, "Network Coded Wireless Architecture", Ph.D Thesis, Department of Electrical Engineering and Computer Science, Massachusetts Institute of Technology, Aug. 29, 2008.

[18] S. Zhang, Y. Zhu, S.-C. Liew, and K. B. Letaief, "Joint Design of Network Coding and Channel Decoding for Wireless Networks", IEEE on Wireless communications and networking Conference WCNC, Kowloon, 2007.

[19] IEEE 802.16 Broadband Wireless Access Working Group, "Channel Models for Fixed Wireless Applications”, IEEE 802.16a-03/01, 27, May, 2003. 